Issued by Sandia National Laboratories, operated for the United States Department of Energy by Sandia Corporation.

NOTICE: This report was prepared as an account of work sponsored by an agency of the United States Government. Neither the United States Government, nor any agency thereof, nor any of their employees, nor any of their contractors, subcontractors, or their employees, make any warranty, express or implied, or assume any legal liability or responsibility for the accuracy, completeness, or usefulness of any information, apparatus, product, or process disclosed, or represent that its use would not infringe privately owned rights. Reference herein to any specific commercial product, process, or service by trade name, trademark, manufacturer, or otherwise, does not necessarily constitute or imply its endorsement, recommendation, or favoring by the United States Government, any agency thereof, or any of their contractors or subcontractors. The views and opinions expressed herein do not necessarily state or reflect those of the United States Government, any agency thereof, or any of their contractors.

Printed in the United States of America. This report has been reproduced directly from the best available copy.

Available to DOE and DOE contractors from

Office of Scientific and Technical Information

P.O. Box 62

Oak Ridge, TN 37831

Prices available from (703) 605-6000

Web site: http://www.ntis.gov/ordering.htm

Available to the public from

National Technical Information Service

U.S. Department of Commerce

5285 Port Royal Rd

Springfield, VA 22161

NTIS price codes

Printed copy: A03

Microfiche copy: A01

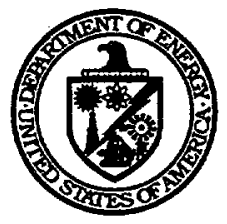




\section{DISCLAIMER}

\section{Portions of this document may be illegible in electronic image products. Images are produced from the best available original document.}




\author{
SAND 99-8255 \\ Unlimited Release \\ Printed October 1999
}

\title{
Using Indicators in Finite Termination Procedures
}

\author{
Pamela J. Williams ${ }^{1}$ \\ Computational Sciences and Mathematics Research \\ Sandia National Laboratories \\ P.O. Box 969, MS 9217 \\ Livermore, CA 94551-0969 \\ pwillia@sandia.gov \\ Amr S. El-Bakry and Richard. A. Tapia ${ }^{2}$ \\ Department of Computational and Applied Mathematics \\ Rice University \\ 6100 Main Street, MS-134 \\ Houston, TX 77005-1892 \\ \{elbakry, rat\}@caam.rice.edu
}

\begin{abstract}
The presence of bounded variables complicates finite termination procedures in interiorpoint methods for linear programming. In our numerical experiments, we found that satisfying the upper bound constraints was the main obstacle to computing an exact solution of a linear program. To prevent the computed solution from violating the bound constraints, one approach

\footnotetext{
${ }^{1}$ Supported by the Mathematical, Information, and Computational Sciences Division subprogram of the Office of Computational and Technology Research, United States Department of Energy, and performed at Sandia National Laboratories operated for the United States Department of Energy under contract no. DE-AC0494AL85000.

${ }^{2}$ The authors were supported in part by DOE DEFG03-93ER15178 and the NSF Cooperative Agreement No. CCR-9120008.
} 
incorporates nearest bound information into a projection model through an affine scaling transformation. This works well in practice but may introduce ill-conditioning due to the potential presence of infinitesimal weights, particularly for variables near a bound.

In this paper, we investigate the role of Tapia indicators in finite termination procedures. Using Tapia indicators, we identify variables in the active set, remove them from the subproblem, and solve a lower dimensional projection problem. Numerical evidence suggests that using Tapia indicators to identify variables in the active set in tandem with an affine scaling transformation results in the fewest iterations needed to compute an exact solution of a linear program. 


\section{Contents}

1 Introduction $\quad 6$

1.1 The Linear Programming Problem . . . . . . . . . . . . . . . . . . . 6

1.2 Algorithmic Framework . . . . . . . . . . . . . . . . 7

2 Background $\quad 9$

3 Mathematical Models $\quad 10$

4 Identification of the Active Set $\quad 11$

5 Computational Results $\quad 13$

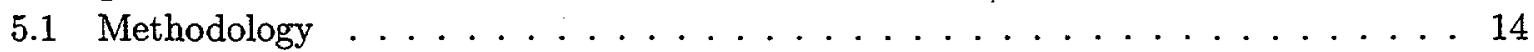

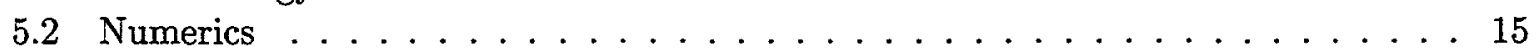

6 Concluding Remarks $\quad 17$ 


\section{Introduction}

We consider one class of finite termination procedures called optimal face identification methods. Optimal face identification methods identify the face upon which the objective function attains its optimal value. The optimal face is uniquely defined by the active set, the set of variables which are at a bound at the solution. Once the active set has been identified, the exact solution of a linear program can be obtained by computing an interior feasible point on the optimal face. For a survey of optimal face identification methods, see Williams, El-Bakry, and Tapia [32].

Adding optimal face identification methods to the interior-point framework can lead to computational savings and highly accurate solutions. Moreover, a point on the optimal face can be used to generate an optimal basic solution in strongly polynomial time, see for example Megiddo [18], Bixby and Saltzman [4], Andersen and Ye [2], and Andersen [3]. Knowledge of the optimal face in sensitivity analysis in the context of interior-point methods was assumed by Adler and Monteiro [1]; Monteiro and Mehrotra [22]; Jansen, Roos, and Terlaky [13, 14]; Jansen, Roos. Terlaky, and Vial [15]; and Greenberg [10, 11].

In this paper, we predict the active set of a linear program and remove the corresponding variables from the optimal face identification problem. We compare the efficiency of this approach with the incorporation of bound information through an affine scaling transformation.

\subsection{The Linear Programming Problem}

We consider linear programs of the form

$$
\begin{array}{ll}
\operatorname{minimize} & c^{T} x \\
\text { subject to } & A x=b \\
& l \leq x \leq u
\end{array}
$$

where $c, x \in \mathbf{R}^{n}, b \in \mathbf{R}^{m}, A \in \mathbf{R}^{m \times n}(m \leq n)$ and $A$ has full rank $m$. The vector $l \in \mathbf{R}^{n}$ represents the vector of lower bounds and $u \in \mathbf{R}^{n}$ represents the vector of upper bounds for the vector $x$. Without loss of generality, we assume all the variables have lower bounds of zero and finite upper bounds. Problem (1) written in standard form is

$$
\begin{array}{ll}
\operatorname{minimize} & c^{T} x \\
\text { subject to } & A x=b, \\
& x+s=u, \\
& x, s \geq 0,
\end{array}
$$

where $s \in \mathbf{R}^{n}$ is the primal slack vector. The inequality $x \geq 0$ denotes component-wise nonnegativity. 
The Karush-Kuhn-Tucker(KKT) conditions for (2) are

$$
F(x, y, z, s, w)=\left(\begin{array}{c}
A x-b \\
x+s-u \\
A^{T} y+z-w-c \\
X Z e \\
S W e
\end{array}\right)=0, \quad(x, z, s, w) \geq 0
$$

where $X=\operatorname{diag}(x), Z=\operatorname{diag}(z), S=\operatorname{diag}(s), W=\operatorname{diag}(w)$, and $e$ is the $n$-vector of all ones. The vector $y \in \mathbf{R}^{m}$ is the vector of Lagrange multipliers corresponding to the equality constraints, $z \in \mathbf{R}^{n}$ is the Lagrange multiplier vector corresponding to the lower bound constraints, and $w \in \mathbf{R}^{n}$ is the Lagrange multiplier vector corresponding to the upper bound constraints.

The Jacobian of (3) is

$$
F^{\prime}(x, y, z, s, w)=\left(\begin{array}{ccccc}
A & 0 & 0 & 0 & 0 \\
I & 0 & 0 & I & 0 \\
0 & A^{T} & I & -I & 0 \\
Z & 0 & X & 0 & 0 \\
0 & 0 & 0 & W & S
\end{array}\right)
$$

The vectors $x$ and $s$ are feasible for the primal problem if $A x=b, x+s=u$, and $(x, s) \geq 0$. We say that $z$ and $w$ are feasible for the dual problem if there exists $y$ such that $(y, z, w)$ is feasible for the dual constraint, $A^{T} y+z-w=c$. A point $(x, y, z, s, w)$ is said to be strictly feasible if it satisfies $A x=b, x+s=u, A^{T} y+z-w=c$, and $(x, z, s, w)>0$.

We denote the solution set of (3) as

$$
\mathcal{S}=\{(x, y, z, s, w): F(x, y, z, s, w)=0,(x, z, s, w) \geq 0\} .
$$

If a solution satisfies $x+z>0$ and $s+w>0$, in addition to $X Z e=0$ and $S W e=0$, then this solution is said to satisfy the strict complementarity condition or strict complementarity. Given a feasible point $(x, y, z, s, w)$ we see that $\|F(x, y, z, s, w)\|_{1}=x^{T} z+s^{T} w$. It can be shown that the expression $x^{T} z+s^{T} w$ is equal to the duality gap, which vanishes at any solution.

\subsection{Algorithmic Framework}

It is well-known that the Kojima, Mizuno, and Yoshise [16] primal-dual interior-point method for linear programming can be viewed as perturbed and damped Newton's method on the first order optimality conditions. In this section, we describe an infeasible primal-dual Newton interior-point method since we implement our finite termination procedures in an infeasible interior-point method.

Algorithm 1 (Infeasible Primal-Dual Interior-Point Algorithm)

Given $v^{0}=\left(x^{0}, y^{0}, z^{0}, s^{0}, w^{0}\right)$ with $\left(x^{0}, z^{0}, s^{0}, w^{0}\right)>0$, for $k=0,1, \ldots$, do 
1. Choose $\sigma^{k} \in(0,1)$ and set $\mu^{k}=\left(\left(x^{k}\right)^{T} z^{k}+\left(s^{k}\right)^{T} w^{k}\right) / 2 n$.

2. Solve for the step $\Delta v^{k}$

$$
F^{\prime}\left(v^{k}\right) \Delta v^{k}=-F\left(v^{k}\right)+\sigma^{k} \mu \tilde{e} \text { where } \mu \geq 0
$$

3. Choose $\tau^{k} \in(0,1)$ and set $\alpha^{k}=\min \left(1, \tau^{k} \hat{\alpha}^{k}\right)$, where

$$
\hat{\alpha}^{k}=\frac{-1}{\min \left(\left(X^{k}\right)^{-1} \Delta x^{k},\left(Z^{k}\right)^{-1} \Delta z^{k},\left(S^{k}\right)^{-1} \Delta s^{k},\left(W^{k}\right)^{-1} \Delta w^{k}\right)} .
$$

4. Let $v^{k+1}:=v^{k}+\alpha^{k} \Delta v^{k}$.

5. Test for convergence.

In Step $2, \tilde{e}=(0, \ldots, 0,1, \ldots, 1)^{T}$ with $2 n+m$ zero components. The optimality conditions (3) are perturbed so that the Newton direction obtained from the perturbed KKT conditions does not poirt towards the boundary. If $\sigma^{k}=0$ (i.e., no perturbation), global convergence may be precluded. See Proposition 3.1 of Gonzalez-Lima [17] and Tapia [28] for a proof. In Step 4, the Newton steps are damped to maintain strict positivity of the iterates.

For notational convenience, we introduce

$$
\tilde{x}=(x, s) \in \mathbf{R}^{2 n} \quad \text { and } \quad \tilde{z}=(z, w) \in \mathbf{R}^{2 n} .
$$

If $\mathcal{S} \neq \emptyset$, then the relative interior of $\mathcal{S}, \operatorname{ri}(\mathcal{S})$, is nonempty. In this case, the solution set $\mathcal{S}$ has the following structure (see El-Bakry, Tapia, and Zhang [6] for a proof): (i) all points in the relative interior satisfy strict complementarity; (ii) the zero-nonzero pattern of points in the relative interior is invariant. For any $\left(\tilde{x}^{*}, y^{*}, \tilde{z}^{*}\right)$ in the relative interior of the solution set of (2), we define the index sets $\tilde{\mathcal{B}}$ and $\tilde{\mathcal{N}}$ as

$$
\tilde{\mathcal{B}}=\left\{j: \tilde{x}_{j}^{*}>0,1 \leq j \leq 2 n\right\} \text { and } \tilde{\mathcal{N}}=\left\{j: \tilde{x}_{j}^{*}=0,1 \leq j \leq 2 n\right\}
$$

Moreover,

$$
\tilde{\mathcal{B}} \bigcup \tilde{\mathcal{N}}=\{1, \ldots, 2 n\} \text { and } \tilde{\mathcal{B}} \bigcap \tilde{\mathcal{N}}=\emptyset
$$

Thus the sets $\tilde{\mathcal{B}}$ and $\tilde{\mathcal{N}}$ define the optimal partition of the set $\{1,2, \ldots, 2 n\}$. The optimal partition uniquely defines the optimal primal and dual faces.

The optimal primal face of (2) is

$$
\Theta_{p}=\left\{\tilde{x}: A x=b, x+s=u, \tilde{x} \geq 0, \tilde{x}_{j}=0 j \in \tilde{\mathcal{N}}\right\}
$$

and the optimal dual face is

$$
\Theta_{d}=\left\{(y, \tilde{z}): A^{T} y+z-w=c, \tilde{z} \geq 0, \tilde{z}_{j}=0 j \in \tilde{\mathcal{B}}\right\}
$$


In the following sections, for $u \in \mathbf{R}^{n}$, we use the notation

$$
\min u=\min _{1 \leq i \leq n} u_{i}
$$

The cardinality of set $\tilde{\mathcal{B}}$ is denoted by $|\tilde{\mathcal{B}}|$. Unless otherwise specified, $\|\cdot\|$ is the Euclidean norm.

The paper is organized as follows. We provide an historical overview of the optimal face identification problem in Section 2. In Section 3 we describe mathematical models to solve the optimal face identification problem. Indicators and their role in finite termination procedures are discussed in Section 4. We present computational results in Section 5 and concluding remarks in Section 6.

\section{Background}

In 1989, stopping tests to compute optimal solutions in interior-point methods for linear programming were proposed by Gay [9]. While these tests did not constitute a finite termination procedure because the primal and dual optimality checks were iterative methods, they were clearly predecessors of current optimal face identification methods. In 1992 Ye [34] popularized the study of finite termination in interior-point methods for linear programming. He was motivated by the fact that the simplex method for linear programming has the finite termination property and also by research activity in efficient algorithmic termination techniques. Ye [34] established a theoretical base for Gay's tests when they are added to primal-dual interior-point algorithms which generate iteration sequences that converge to strict complementarity solutions. The author proposed an orthogonal projection model to identify the optimal primal and dual faces. Mehrotra and Ye [21] developed a solution technique based on Gaussian elimination to compute an interior feasible point on the optimal primal and dual faces. Previously Tardos [29] used Gaussian elimination to calculate a feasible point on the optimal face of an integer program. Recently, Ye [35] proposed a weighted projection model which incorporates bound information, for the standard linear program with no upper bound constraints. Ye [35] proved for $k$ sufficiently large his method when included in a feasible primal-dual interior-point method computed an exact solution of a linear program in finite time. Williams, El-Bakry, and Tapia [33] extended Ye's weighted projection model and analysis to linear programs with bounded variables.

The following lemma provides a theoretical basis for most finite termination procedures for linear programs of the form

$$
\begin{array}{ll}
\operatorname{minimize} & c^{T} x \\
\text { subject to } & A x=b \\
& x \geq 0
\end{array}
$$


Lemma 2.1 (Güler-Ye [12]) Let $\left\{\left(x^{k}, y^{k}, z^{k}\right)\right\}$ be an iteration sequence generated by an interiorpoint algorithm. Furthermore, let $x^{k}$ and $z^{k}$ satisfy

$$
\frac{\min \left(X^{k} Z^{k} e\right)}{\left(x^{k}\right)^{T} z^{k} / n} \geq \gamma
$$

where $\gamma>0$ and is independent of $k$. Then every limit point of $\left\{\left(x^{k}, z^{k}\right)\right\}$ satisfies the strict complementarity condition.

Lemma 2.1 is sufficient to guarantee that all limit points of the iteration sequence are in the relative interior of the solution set. Since the nonzero-zero pattern of points is invariant in the relative interior, the optimal primal and dual faces of (5) are uniquely defined.

\section{Mathematical Models}

Before describing our proposed modification, we briefly discuss projection models for (5) and define some notation. The projection models for linear program (5) are important because traditionally researchers have not explicitly included upper bound constraints into the subproblem. Instead they treat the upper bounds as side constraints.

We define the index sets $\mathcal{B}$ and $\mathcal{N}$ as

$$
\mathcal{B}=\left\{j: x_{j}^{*}>0,1 \leq j \leq n\right\} \text { and } \mathcal{N}=\left\{j: x_{j}^{*}=0,1 \leq j \leq n\right\} .
$$

The index sets $\mathcal{B}$ and $\mathcal{N}$ define the optimal partition of (5). The columns of $A$ corresponding to the indices of $\mathcal{B}$ comprise the matrix $A_{\mathcal{B}}$. The matrix $A_{\mathcal{N}}$ is formed in an similar manner. The vector $x_{i 3}$ represents the components of the vector $x$ whose indices are in $\mathcal{B}$.

Ye [35] was the first to incorporate bound information into the optimal face identification model via an affine scaling transformation of the subproblem. For the standard linear program (5), Ye posed two projection problems

$$
\begin{array}{ll}
\min & \frac{1}{2}\left\|\left(X_{\mathcal{B}}^{k}\right)^{-1}\left(x_{\mathcal{B}}-x_{\mathcal{B}}^{k}\right)\right\|^{2} \\
\text { s.t. } & A_{\mathcal{B}} x_{\mathcal{B}}=b
\end{array}
$$

and

$$
\begin{array}{ll}
\min & \frac{1}{2}\left\|\left(Z_{\mathcal{N}}^{k}\right)^{-1} A_{\mathcal{N}}^{T}\left(y-y^{k}\right)\right\|^{2} \\
\text { s.t. } & A_{\mathcal{B}}^{T} y=c_{\mathcal{B}},
\end{array}
$$

to find an interior solution on the optimal primal and dual faces, respectively. The primal model restricts movement in components that can least afford to deviate from $x_{\mathcal{B}}^{k}$ by placing large weights on the smaller components. Similarly, the dual model attempts to restrict movement of $z_{\mathcal{N}}^{k}$ components. 
Williams [31] and Williams, El-Bakry, Tapia [33] extended Ye's work to linear programs with bounded variables. The authors proposed the following modified weighted projection model

$$
\begin{array}{ll}
\min & \frac{1}{2}\left\|\left(D^{k}\right)^{-1}\left(x_{\mathcal{B}}-x_{\mathcal{B}}^{k}\right)\right\|^{2} \\
\text { s.t. } & A_{\mathcal{B}} x_{\mathcal{B}}=b,
\end{array}
$$

where

$$
d_{j j}^{k}=\min \left(x_{j}^{k}, u_{j}-x_{j}^{k}\right) \quad \text { for } j \in \mathcal{B},
$$

as the optimal primal face identification problem and

$$
\min _{y} \frac{1}{2}\left\|D^{k}\left(A_{\mathcal{B}}^{T} y-w_{\mathcal{B}}-c_{\mathcal{B}}\right)\right\|^{2}
$$

as the optimal dual face identification problem. Given formulation (11), one matrix factorization is needed to solve both the optimal primal and dual face identification problems as opposed to the two matrix factorizations required by (7) and (8). If no upper bounds exist, problem (9) reduces to problem (7).

Weighting the objective function by $D^{k}$ penalizes the movement of the variables in the direction of their nearest bound. Therefore, if $x_{j}^{k}$ for $j \in \mathcal{B}$ is close to its upper bound, the weight in (10) prevents the $j$ th component of the solution vector $x_{\mathcal{B}}$ from violating its upper bound as well as its lower bound, which is the desired result. The use of weights to prevent bound violations is not novel. Plantenga [23], for example, proposed scaling the quadratic subproblem of a sequential quadratic programming algorithm for bound and equality constrained optimization using trust regions by $D^{k}$ to prevent bound violations.

The affine scaling transformation in (9) has the following drawback. If any variable is 'close' to its upper bound, the scale $D^{k}$ has the potential of introducing ill-conditioning into the subproblem. We want to minimize this possibility by removing these problematic variables from the subproblem. The difficulty arises in defining 'closeness' of a variable to its upper bound. The next section addresses this issue.

\section{Identification of the Active Set}

The term indicator denotes a function that identifies active constraints at the solution of a constrained optimization problem, see Tapia [27] and El-Bakry [5]. Commonly used indicators include variables as indicators, the primal-dual indicator, and the Tapia indicator. Recently, Facchinei, Fischer, and Kanzow [8] proposed an indicator based on growth functions to identify the active set of column sufficient linear complementarity problems. A similar indicator for general nonlinear programs was introduced in Facchinei, Fischer, and Kanzow [7].

An extensive numerical comparison of indicators as stopping criterion can be found in de Vreede [30]. For a thorough study of indicator theory, see El-Bakry [5] and El-Bakry, Tapia, and Zhang [6]. 
Tapia [27] used the following indicators to determine the active set in nonlinear constrained optimization problems. The Tapia indicators are

$$
T_{p}\left(x_{j}^{k}\right)=\frac{x_{j}^{k+1}}{x_{j}^{k}} \quad \text { and } \quad T_{d}\left(z_{j}^{k}\right)=\frac{z_{j}^{k+1}}{z_{j}^{k}}
$$

where $x_{j}^{k+1}=x_{j}^{k}+\beta^{k} \Delta x_{j}^{k}$ and $z_{j}^{k+1}=z_{j}^{k}+\beta^{k} \Delta z_{j}^{k}$. In [6] El-Bakry, Tapia, and Zhang showed the Tapia indicators have a $0-1$ separation property and converge $R$-superlinearly to their terminal values.

It is well-known that the Tapia indicators are an effective computational tool for identifying the active set of problem (5), which can lead to reduction of problem size and computational savings. Because of the efficacy of the Tapia indicator in identifying variables which are zero on the solution set, it is the natural choice to identify variables at their upper bounds. Unfortunately the indicator, $T_{p}\left(x_{j}^{k}\right)$, does not differentiate between variables that are at their upper bounds and those which are strictly between their lower and upper bounds. Other indicators such as variables as indicators and the logarithmic Tapia indicators predict variables at the upper bounds with varying success. However, this drawback is easily remedied.

Let's consider the upper bound constraint

$$
x+s=u \text {. }
$$

We see that as $x_{j}^{k} \rightarrow u_{j}$, then $s_{j}^{k} \rightarrow 0$. Therefore determining variables at their upper bounds is the same as identifying which primal slack variables are zero.

The Tapia indicators for the primal slack variable, $s$, and its corresponding Lagrange multiplier, $w$, are

$$
T\left(s_{j}^{k}\right)=\frac{s_{j}^{k+1}}{s_{j}^{k}} \quad \text { and } \quad T\left(w_{j}^{k}\right)=\frac{w_{j}^{k+1}}{w_{j}^{k}},
$$

where $s_{j}^{k+1}=s_{j}^{k}+\beta^{k} \Delta s_{j}^{k}$ and $w_{j}^{k+1}=w_{j}^{k}+\beta^{k} \Delta w_{j}^{k}$.

We use the notation

$$
\mathcal{U}=\left\{i: s_{i}=0,1 \leq i \leq n\right\}
$$

to denote the primal slack variables which are zero at the solution of (2) and correspondingly the $x$ variables which equal their upper bounds.

The following proposition shows that the Tapia indicators for the slack variables and the corresponding Lagrange multipliers for the upper bound constraints have a 0-1 separation property. For numerical experimentation, the 0-1 convergence limits of the Tapia indicators provide us with a theoretical basis for an indicator threshold value to use in active set identification.

Proposition 4.1 Consider a sequence of iterates $\left\{\left(x^{k}, y^{k}, z^{k}, s^{k}, w^{k}\right)\right\}$ generated by Algorithm 1. Assume 
1. $\left(x^{k}\right)^{T} z^{k}+\left(s^{k}\right)^{T} w^{k} \rightarrow 0 ;$

2. $\min \left(X^{k} Z^{k} e, S^{k} W^{k} e\right) \geq \gamma \mu^{k}$, for all $k$ and for some $\gamma \in(0,1)$;

3. The algorithmic parameters are chosen such that

$$
\sigma^{k} \rightarrow 0 \text { and } \tau^{k} \rightarrow 1
$$

Then for $j=1, \ldots, n$

$$
\begin{array}{r}
\lim _{k \rightarrow \infty} \frac{s_{j}^{k+1}}{s_{j}^{k}}= \begin{cases}0 & j \in \mathcal{U} \\
1 & j \notin \mathcal{U}\end{cases} \\
\lim _{k \rightarrow \infty}\left(1-\frac{w_{j}^{k+1}}{w_{j}^{k}}\right)= \begin{cases}0 & j \in \mathcal{U} \\
1 & j \notin \mathcal{U}\end{cases}
\end{array}
$$

where $s^{k+1}=s^{k}+\beta^{k} \Delta s^{k}$ and $w^{k+1}=w^{k}+\beta^{k} \Delta w^{k}$ for any $\beta^{k} \in\left[\alpha^{k}, 1\right]$ with $\alpha^{k}$ given in step 3 of Algorithm 1.

An alternative is to apply the Tapia indicator directly to the vector $\tilde{x}$, which includes the primal slack vector. We can then extract bound information from the terminal value of $T_{p}\left(\tilde{x}_{j}\right)$, but for our purposes it is more convenient to consider the slack vector separately.

In the following section, we describe numerical experiments performed to test the effectiveness of the proposed optimal face identification models in computing an exact solution of a linear program. We measure effectiveness in terms of the number of projection attempts that are needed to compute an exact solution of a linear program.

\section{Computational Results}

In our numerical experiments, we used the LIPSOL - Linear programming Interior-Point SOLver- package developed under the MATLAB ${ }^{3}$ environment. The software package, written by Zhang [36], implements an infeasible primal-dual predictor-corrector interior-point method. We selected 35 problems with upper bounds from the netlib suite of linear programming problems as our test set.

The initial matrix is scaled in an attempt to achieve row/column equilibration. Preprocessing deletes fixed variables, deletes zero rows and columns from the matrix $A$, solves equations of one variable, and shifts nonzero lower bounds to zero.

\footnotetext{
${ }^{3}$ MATLAB is a registered trademark of The MathWorks, Inc.
} 


\subsection{Methodology}

We first attempt to compute an exact solution of a linear program, when

$$
\frac{\left|c^{T} x^{k}-\left(b^{T} y^{k}-u^{T} w^{k}\right)\right|}{1+\left|b^{T} y^{k}-u^{T} w^{k}\right|} \leq 10^{-8}
$$

Another option is to project when maximum relative error,

$$
\max \left(\frac{\|A x-b\|}{1+\|b\|}, \frac{\left\|A^{T} y+z-w-c\right\|}{1+\|c\|}, \frac{\left|c^{T} x-\left(b^{T} y-u^{T} w\right)\right|}{1+\left|b^{T} y-u^{T} w\right|}\right) \leq 10^{-8}
$$

Mehrotra and Ye in [21], Mehrotra in ([19, 20]), as well as Andersen and Ye [2] used the Tapia indicators to identify the optimal partition. Specifically, they defined

$$
\mathcal{B}^{k}=\left\{j: \frac{\left|\Delta x_{j}^{k}\right|}{x_{j}^{k}} \leq \frac{\left|\Delta z_{j}^{k}\right|}{z_{j}^{k}}\right\}
$$

Mehrotra and Ye [21] proved that when $\mathcal{B}^{k}$ was defined as in (12) the optimal partition could be identified in finite time for algorithms that generate iteration sequences that satisfy centrality measure (6). For numerical experiments, the authors used the Tapia indicators and variables as indicators in tandem.

Following their philosophy, we let

$$
\mathcal{B}^{k}=\left\{j: z_{j}^{k} \leq 10^{-14} \quad \text { or } \quad\left|\Delta x_{j}^{k}\right| / x_{j}^{k} \leq\left|\Delta z_{j}^{k}\right| / z_{j}^{k}\right\} .
$$

Similarly,

$$
\mathcal{U}^{k}=\left\{j: s_{j}^{k} \leq 10^{-14} \text { or }\left|\Delta w_{j}^{k}\right| / w_{j}^{k} \leq\left|\Delta s_{j}^{k}\right| / s_{j}^{k}\right\} .
$$

We then redefine $\mathcal{B}^{k}$ as

$$
\mathcal{B}^{k}:=\mathcal{B}^{k} \backslash \mathcal{U}^{k}
$$

This step is necessary since the set in (13) may contain indices of variables at their upper bounds.

After setting

$$
x_{\mathcal{U}}=u_{\mathcal{U}}, \quad x_{\mathcal{N}}=0,
$$

we solve

$$
\begin{array}{ll}
\min & \frac{1}{2}\left\|\left(D^{k}\right)^{-1}\left(x_{\mathcal{B}}-x_{\mathcal{B}}^{k}\right)\right\|^{2} \\
\text { s.t. } & A_{\mathcal{B}} x_{\mathcal{B}}=b-A_{\mathcal{U}} x_{\mathcal{U}}
\end{array}
$$

and

$$
\min _{y} \frac{1}{2}\left\|D^{k}\left(A_{\mathcal{B}}^{T} y-w_{\mathcal{B}}-c_{\mathcal{B}}\right)\right\|^{2}
$$


for $x_{\mathcal{B}}$ and $y$. We then update $z, s, w$ as follows.

Set $s=u-x$ and $\delta=c-A^{T} y$, then

$$
z_{j}=\left\{\begin{array}{ll}
0 & \text { if } \delta_{j}<0 \\
\delta_{j} & \text { else }
\end{array} \text { and } w_{j}= \begin{cases}-\delta_{j} & \text { if } \delta_{j}<0 \\
0 & \text { else. }\end{cases}\right.
$$

The update formula for the dual variables was used by Resende and Veiga in [25], Resende, Tsuchiya, and Veiga [26] and Portugal, Resende, Veiga, and Judice [24] to generate feasible dual variables in network flow problems.

If the computed solution is complementary and

$$
\max \left(\frac{\|A x-b\|}{1+\|b\|}, \frac{\left\|A^{T} y+z-w-c\right\|}{1+\|c\|}, \frac{\left|c^{T} x-\left(b^{T} y-u^{T} w\right)\right|}{1+\left|b^{T} y-u^{T} w\right|}\right) \leq 10^{-11}
$$

the algorithm terminates with an exact solution of a linear program. If not, the procedure is repeated at the next interior-point iteration.

We allow a maximum of six projection attempts to compute an exact solution.

\subsection{Numerics}

Table 1 illustrates problem size reduction, one of the benefits of identifying variables which are at their upper bounds. For problem fit1d, removal of variables at their bounds transforms the linear system from an undetermined system to a square system.

Table 1: Problem Size Reduction

\begin{tabular}{|c|c|c|c|}
\hline Problem & $\left|\mathcal{B}^{k}\right|$ & $\left|\mathcal{U}^{k}\right|$ & $\left|\mathcal{B}^{k} \backslash \mathcal{U}^{k}\right|$ \\
\hline boeing2 & 123 & 6 & 117 \\
\hline finnis & 435 & 17 & 418 \\
\hline fit1d & 377 & 353 & 24 \\
\hline greenbeb & 1456 & 194 & 1262 \\
\hline pilot4 & 611 & 247 & 364 \\
\hline pilotnov & 2114 & 1 & 2113 \\
\hline recipe & 147 & 8 & 139 \\
\hline seba & 442 & 3 & 439 \\
\hline vtpbase & 135 & 32 & 103 \\
\hline
\end{tabular}

We compare three projection models to compute an exact solution of a linear program. The first one is the orthogonal projection model where $D^{k}=I$, the second is Ye's weighted projection method (7) and the third is the modified weighted projection. The first two models 
were developed by Ye [34, 35]. Column 1 of Tables 2 and 3 gives the number of failed calls (misses) to the finite termination procedure before the optimal face identification problem was solved to the desired accuracy. We consider a call a failure if the procedure does not generate a positive solution that satisfies the given feasibility tolerances. The second column gives the number of problems solved by the orthogonal projection model for the given number of misses. The third column contains the computational results of Ye's weighted projection model and the fourth column the modified weighted projection.

Table 2: Variables at Upper Bounds Not Removed

\begin{tabular}{|c|c|c|c|}
\hline & \multicolumn{3}{|c|}{ Models } \\
\hline \# of misses & Orthogonal & Weighted & Modified Weighted \\
\hline 0 & 13 & 15 & 19 \\
\hline 1 & 13 & 12 & 11 \\
\hline 2 & 2 & 2 & 4 \\
\hline 3 & 2 & 3 & 1 \\
\hline 4 & 2 & 0 & 0 \\
\hline 5 & 1 & 1 & 0 \\
\hline more than 5 & 2 & 2 & 0 \\
\hline
\end{tabular}

Table 3: Variables at Bounds Removed

\begin{tabular}{|c|c|c|c|}
\hline & \multicolumn{3}{|c|}{ Models } \\
\hline \# of misses & Orthogonal & Weighted & Modified Weighted \\
\hline 0 & 21 & 21 & 22 \\
\hline 1 & 9 & 8 & 8 \\
\hline 2 & 3 & 4 & 3 \\
\hline 3 & 2 & 2 & 2 \\
\hline
\end{tabular}

Identifying the variables at their upper bounds plays a crucial role in finite termination procedures. The orthogonal projection model computes an exact solution of a linear program with $60 \%$ fewer misses on the first attempt; the weighted projection model has approximately $40 \%$ fewer misses. The reduction in projection attempts represents a substantial savings in computational expense.

To further demonstrate the importance of identifying and removing variables at their upper bounds, in Table 4 we consider three problems, etamacro, greenbea, nesm, for which either 6 projection attempts were needed or the optimal face identification procedure failed to compute 
an exact solution. Removing the variables at the upper bounds produces a dramatic decrease in the projection attempts. In particular, the problem greenbea requires 5 fewer projections.

Table 4: Orthogonal Projection Model

\begin{tabular}{|c|c|c|}
\hline & \multicolumn{2}{|c|}{ Misses } \\
\hline problem & Variables at Upper Bounds not Identified & Identified \\
\hline etamacro & 5 & 3 \\
\hline greenbea & $>5$ & 0 \\
\hline nesm & $>5$ & 2 \\
\hline
\end{tabular}

It is important to point out that for these three problems the number of misses with variables at their upper bounds identified is the same as the modified weighted projection model without variables at upper bounds identified. This suggests that the modified scale simulates removal of variables at their upper bounds from the problem.

\section{Concluding Remarks}

Using Tapia indicators to identify variables at their upper bounds increases the efficiency of optimal face identification procedures. For best results we recommend the implementation of a finite termination procedure which includes Tapia indicators as well as an affine scale transformation which incorporates bound constraints.

\section{Acknowledgments}

The authors would like to thank Yin Zhang for discussions on material related to this paper.

\section{References}

[1] I. Adler and R. Monteiro. A Geometric View of Parametric Linear Programming. Algorithmica, 8:161-176, 1992.

[2] E. Andersen and Y. Ye. Combining interior-point and pivoting algorithms for linear programming. Technical Report, Department of Management Sciences, University of Iowa, 1994.

[3] E. Andersen. On exploiting problem structure in a basis identification procedure for linear programming. Technical Report, Department of Management, Odense University, Denmark, 1996. 
[4] R. Bixby and M. Saltzman. Recovering an Optimal LP Basis from an Interior-Point Solution. Technical Report, TR91-32, Department of Mathematical Sciences, Rice University, 1991.

[5] A. El-Bakry. On the role of indicators in identifying zero variables in linear programming. PhD thesis, Department of Mathematical Sciences, Rice University, 1991.

[6] A. El-Bakry, R. Tapia, and Y. Zhang. A study of indicators for identifying zero variables in interior-point methods. SIAM Review, 36:45-72, 1994.

[7] F. Facchinei, A. Fischer, and C. Kanzow. On the accurate identification of active constraints. SIAM Journal on Optimization, 9(1):14-32, 1999.

[8] F. Facchinei, A. Fischer, and C. Kanzow. On the Identification of Zero Variables in an Interior-Point Framework. Mathematical Programming Technical Report 98-06, Computer Sciences Department, University of Wisconsin-Madison, May 1998.

[9] D. Gay. Stopping tests that compute optimal solutions for interior-point linear programming algorithms. In S. Gómez, J. Hennart, and R. Tapia, eds., Advances in Numerical Partial Differential Equations and Optimization: Proceedings of the Fifth Mexico-United States Workshop, pages 17-42. Society for Industrial and Applied Mathematics, Philadelphia, PA, 1991.

[10] H. Greenberg. Rim Sensitivity Analysis from an Interior Solution. CCM N0. 86, Center for Computational Mathematics, Mathematics Department, University of Colorado at Denver, 1996.

[11] H. Greenberg. Matrix Sensitivity Analysis from an Interior Solution of a Linear Program. CCM N0. 104, Center for Computational Mathematics, Mathematics Department, University of Colorado at Denver, 1997.

[12] O. Güler and Y. Ye. Convergence behavior of interior-point algorithms. Mathematical Programming, 60:215-228, 1993.

[13] B. Jansen, C. Roos, and T. Terlaky. Sensitivity analysis in linear programming: just be careful! European Journal of Operations Research, 101:15-28, 1997.

[14] B. Jansen, C. Roos, and T. Terlaky. An Interior-Point Approach to Postoptimal and Parametric Analysis in Linear Programming. Report No. 92-21, Faculteit der Technische Wiskunde en Informatica, Technische Universiteit Delft, The Netherlands.

[15] B. Jansen, C. Roos, T. Terlaky, and J.-Ph. Vial. Interior-Point Methodology for Linear Programming: Duality, Sensitivity Analysis and Computation Aspects. In K. Frauendorfer, H. Glavitsch, and R. Bacher, eds., Optimization in Planning and Operation of Electric Power Systems, pages 57-123. Physica Berlag, 1993. 
[16] M. Kojima, S. Mizuno, and A. Yoshise. A primal-dual interior-point method for linear programming. In N. Megiddo, ed., Progress in Mathematical Programming, Interior Point and Related Methods, pages 29-47. Springer-Verlag, New York, NY, 1989.

[17] M. Gonzalez-Lima. Effective Computation of the Analytic Center of the Solution Set in Linear Programming Using Primal-Dual Interior-Point Methods. PhD thesis, Department of Computational and Applied Mathematics, Rice University, 1994.

[18] N. Megiddo. On Finding Primal- and Dual-Optimal Bases. ORSA Journal on Computing, $3: 1,63-65,1991$.

[19] S. Mehrotra. On Finding a Vertex Solution Using Interior-Point Methods. Linear Algebra and its Applications, 152:233-253, 1991.

[20] S. Mehrotra. Finite Termination and Superlinear Convergence in Primal-Dual Methods. Technical Report TR91-13, Dept. of Industrial Engineering and Management Sciences, Northwestern University, 1991.

[21] S. Mehrotra and Y. Ye. Finding an Interior Point in the Optimal Face of Linear Programs. Mathematical Programming, 62:497-515, 1993.

[22] R. Monteiro and S. Mehrotra. A General Parametric Analysis Approach and its Implication to Sensitivity Analysis in Interior-Point Methods. Mathematical Programming, 72: 65-82, 1996.

[23] T. Plantenga. A Trust Region Method for Nonlinear Programming Based on Primal Interior-Point Techniques. Technical Report, 1997.

[24] L. Portugal, M. Resende, G. Veiga, and J. Judice. A Truncated Primal-Infeasible DualFeasible Network Interior-Point Method. Technical Report, Information Sciences Research Center, AT\&T Bell Laboratories, 1994.

[25] M. Resende and G. Veiga. An Efficient Implementation of a Network Interior-Point Method. In D. Johnson and C. McGeoch, eds., Network Flows and Matching: First DIMACS Implementation Challenge, Volume 12 of DIMACS Series in Discrete Mathematics and Theoretical Computer Science, pages 299-348. American Mathematical Society, 1993.

[26] M. Resende, T. Tsuchiya, and G. Veiga. Identifying the Optimal Face of a Network Linear Program with a Globally Convergent Interior-Point Method. In W. Hager, D. Hearn, and P. Pardalos, eds., Large-Scale Optimization: State of the Art, pages 362-387. Kluwer Academic Publishers, 1994.

[27] R. Tapia. On the role of slack variables in quasi-Newton methods for constrained optimization. In L.C.W. Dixon and G.P. Szegö, eds., Numerical Optimization of Dynamic Systems, pages 235-246. North Holland, 1980. 
[28] R. Tapia. On the Fundamental Role of Interior-Point Methodology in Constrained Optimization. Technical Report TR97-09, Department of Computational and Applied Mathematics, Rice University, 1997.

[29] E. Tardos. A Strongly Polynomial Algorithm to Solve Combinatorial Linear Programs. Operations Research, 36(2):250-256, 1986.

[30] F. de Vreede. Indicators as stopping criteria in interior-point methods in linear programming. Master's thesis, The Netherlands, 1993.

[31] P. Williams. Effective Finite Termination Procedures in Interior-Point Methods for Linear Programming. Ph.D. thesis, Department of Computational and Applied Mathematics, Rice University, 1998.

[32] P. Williams, A. El-Bakry, and R. Tapia. Computing an Exact Solution in Interior-Point Methods for Linear Programming. (to appear in African-Americans in Mathematics II: Proceedings of the Fourth Conference for African-American Researchers in the Mathematical Sciences).

[33] P. Williams, A. El-Bakry, and R. Tapia. The Role of Affine Scaling Transformations in Optimal Face Identification Methods. Working Paper. Department of Computational and Applied Mathematics, Rice University.

[34] Y. Ye. On the Finite Convergence of Interior-Point Algorithms for Linear Programming. Mathematical Programming( Series B), 57:325-335,1992.

[35] Y. Ye. Interior-Point Algorithm: Theory and Analysis, John Wiley \& Sons, New York, New York, 1997.

[36] Y. Zhang. LIPSOL beta version 2.1. Department of Mathematics and Statistics, University of Maryland Baltimore County, 1995. 
UNLIMITED RELEASE

INITIAL DISTRIBUTION:

5 Dr. Richard A. Tapia

Department of Computational

\& Applied Mathematics

MS 134

Rice University

6100 Main Street

Houston, TX 77005-1892

5 Dr. Amr S. El-Bakry

Department of Computational

\& Applied Mathematics

MS 134

Rice University

6100 Main Street

Houston, TX 77005-1892

1 Dr. Tamas Terlaky

Department of Statistics,

Probability, and Operations Research

Faculty of Technical Mathematics

\& Informatics

Delft University of Technology

P.O. Box 5031

2600 GA Delft

The Netherlands

1 MS 9001 M. E. John, 8000

Attn: R. C. Wayne, 2200

M.E. John, 8100

L.A. West, 8200

W.J. McLean, 8300

D. R. Henson, 8400

P.N. Smith, 8500

P.E. Brewer, 8600

T.M. Dyer, 8700

L.A. Hiles, 8800

M. Rogers, 8401 
1 MS 9003 K. E. Washington, 8900

1 MS 9003 D. L. Crawford, 9900

1 MS 9011 P.W. Dean, 8903

1 MS 9011 B. Hess, 8910

1 MS 9011 P. Nielan, 8920

1 MS 9011 N. Berry, 8920

1 MS 9012 R. Trechter, 8930

1 MS 9037 J. Berry, 8930-1

1 MS 9019 B. A. Maxwell, 8940

$5 \quad$ MS 9217 J. C. Meza, 8950

1 MS 9019 J. A. Larson, 8970

1 MS $9012 \mathrm{~K}$. Hughes, 8990

1 MS 9217 P. Boggs, 8950

1 MS 9217 P. Hough, 8950

1 MS 9217 T. Kolda, 8950

1 MS 9217 K. Long, 8950

$5 \quad$ MS 9217 P. Williams, 8950

1 MS 1110 D. Womble, 9222

3 MS 9018 Central Technical Files, 8940-2

1 MS 0899 Technical Library, 4916

1 MS 9021 Technical Communications

Department, 8815/Technical Library MS 0899, 4916

1 MS 9021 Technical Communications

Department, 8815 For DOE/OSTI 